\title{
ANÁLISE DA MOVIMENTAÇÃO DE PEDESTRES ATRAVÉS DE SIMULAÇÃO COMPUTACIONAL
}

\author{
Ivana Righetto Moser \\ Universidade Federal de Santa Catarina \\ Campus UFSC - Trindade - PósARQ/CTC - Florianópolis - SC \\ irighetto@yahoo.com.br \\ João Carlos Souza \\ Universidade Federal de Santa Catarina \\ Campus UFSC - Trindade - PósARQ/CTC - Florianópolis - SC \\ joao.carlos@ufsc.br \\ Patricia Kuwer \\ Universidade Federal de Santa Catarina \\ Campus UFSC - Trindade - PósARQ/CTC - Florianópolis - SC \\ patriciakuwer@gmail.com
}

\begin{abstract}
RESUMO
O objetivo desta pesquisa foi estudar a movimentação de pedestres em situação de evacuação emergencial. Foram avaliados simuladores que utilizam o comportamento humano como variável e foi aplicado o software PTV Viswalk, que se entende como a melhor opção para esta pesquisa. Conjuntamente são discutidos os conceitos referentes ao estudo do tráfego de pedestres, a modelagem e a simulação com humanos virtuais e modelos computacionais de evacuação de emergência de pessoas. Como resultado das simulações foram observados: gargalos, interações entre pessoas, variações de velocidade durante o deslocamento, além de aglomerações. Entende-se que a adoção de método computacional pode auxiliar no aumento de segurança dos pedestres, pois trata-se de uma ferramenta que possibilita visualizar o que ocorre em uma possível situação de emergência e consequentemente, permite uma melhor análise dos pontos críticos em uma edificação.
\end{abstract}

Palavra-chave: Movimentação de pedestres; Evacuação emergencial; Comportamento humano; Simulação computacional; Segurança em edifícios.

\begin{abstract}
The objective of this research was to study the movement of pedestrians in emergency evacuations. Were evaluated computational simulators that use human behavior as a variable and the software PTV Viswalk was applied, wich is understood as the best option for this research. Together the concepts related to the study of pedestrian traffic, modelling and simulation with virtual humans and computational models of emergency evacuation of people are discussed. As result of the simulations were observed: bottlenecks, interactions between
\end{abstract}


people, variations in speed during the displacement, besides agglomerations. It is understood that the adoption of computational method can help increase the safety of pedestrian, because it is a tool that allows to visualize what happens in a possible emergency situation and, consequently, allows a better analysis of critical points in a building.

Keywords: Pedestrian movement; Emergency evacuation; Human behavior; Computer simulation; Security in buildings.

\section{Como Citar:}

MOSER, Ivana Righetto; SOUZA, João Carlos; KUWER, Patricia. Análise da movimentação de pedestres através de simulação computacional. In: SIMPÓSIO DE PESQUISA OPERACIONAL E LOGÍSTICA DA MARINHA, 19., 2019, Rio de Janeiro, RJ. Anais [...]. Rio de Janeiro: Centro de Análises de Sistemas Navais, 2019.

\section{INTRODUÇÃ̃O}

O potencial letal de movimentação em edificações com um grande número de pessoas é visualizado através de exemplos de grandes incidentes que ocorrem em uma ampla variedade de locais e diferentes circunstâncias. O Departamento de Iniciativa Humanitária da Universidade Harvard, nos Estados Unidos, juntamente com um grupo de pesquisadores, reuniu notícias de eventos envolvendo multidões em que pessoas haviam sido mortas ou gravemente feridas em razão de tumultos. Os pesquisadores perceberam que o número de casos está longe de ser desprezível e concluíram que eles se tornaram mais frequentes nas últimas décadas [1].

Uma vez iniciado um evento atípico dentro de uma edificação com aglomeração de pessoas, é difícil retomar o controle da situação. O movimento de um grande número de pessoas precisa ser planejado com cautela, a fim de que sejam adotadas medidas protetivas, tal como velocidades de saída seguras, evitando estrangulamentos, curvas ou obstáculos ao sistema, que podem ocasionar aglomeração ou lentidão em alguns pontos.

Pesquisas sobre rotas de fuga e saídas de emergência, com efetivo abandono do local, vêm sendo desenvolvidas por pesquisadores de vários países do mundo, como Japão [2], Países Baixos [3] e Brasil [4]. O comportamento humano em situações de risco de incêndio, ou em outras situações de risco, é amplamente estudado por diversas áreas como psicologia [5;6] e engenharia de tráfego [7;8]. Destaca-se, também, as pesquisas experimentais que se utilizam de modelos e ensaios [3;9]. O comportamento em situação de risco varia de pessoa para pessoa e o estresse é muito particular e pouco está ligado à idade ou força do indivíduo.

Modelos computacionais de simulação de evacuação são ferramentas amplamente utilizadas para avaliação da segurança das pessoas em um edifício ou locais de aglomeração e estes associados à análise do comportamento humano, faz com que os resultados do esvaziamento emergencial sejam mais corretos e conclusivos. Esse artigo tem o objetivo de estudar a movimentação de pedestres durante evacuação de emergência.

\section{O ESTUDO DE TRÁFEGO DE PEDESTRES}

O fluxo de tráfego de pedestres é definido por "uma massa de pessoas que se desloca simultaneamente e indiretamente ao longo de uma rota mútua” [9]. Neste conceito, segundo os autores, devem ser levados em conta fatores como diferentes tipos de movimento, estado psicológico e físico, diferentes tipos de rotas, entre outros.

As primeiras pesquisas científicas de tráfego de pedestres iniciaram na década de 1930, por meio de parâmetros de fluxos e, em seguida, passaram a ser realizados 
experimentos práticos de observação. Das observações visuais, passou-se a adotar a gravação por imagens e, consequentemente, deu-se o aperfeiçoamento dos estudos, com métodos de melhorias das filmagens que evitavam distorções por perspectivas, dentre outros. Obtiveramse, assim, resultados mais objetivos e em maior número [9]. Helbing et al. [7], por exemplo, fizeram observações por meio de gravações e puderam caracterizar uma regularidade no movimento de pedestres, em situações normais, quando observados em câmera-rápida ou time-lapse. Em seus resultados obtiveram que as pessoas tendem a manter uma distância entre outras pessoas e barreiras e possuem aversão em desviar do caminho desejado. Kholshevnikov e Samoshin [9], por sua vez, alertam que o uso incorreto de métodos de observação pode levar a resultados com porcentagem significativa de erros e que o uso incorreto da estatística pode levar a "milagres".

Os cálculos utilizados em tráfego de veículos são também utilizados para o estudo em que o escopo é o deslocamento de pedestres. Os modelos de comportamento coletivo são do tipo macroscópico, que avaliam as correntes de tráfego de pessoas e como se comportam; microscópico, que avaliam as interações entre indivíduos, ou seja, dois indivíduos dentro de um fluxo de pedestres; e a mesoscópica ou cinética, utilizada para analisar agrupamentos formados por pessoas em ruas, praças, terminais de transporte, ou em outro lugar que se deseja estudar. Dentre as vantagens e desvantagens de cada abordagem, pode-se citar: nos modelos macroscópicos podem-se aplicar modelos hidrodinâmicos que têm simplicidade computacional. Contudo, deve-se introduzir uma relação empírica entre a velocidade e a densidade, fugindo da precisão do fluxo real. No modelo microscópico é possível uma observação mais cuidadosa de cada pedestre, porém, isso demanda um grande esforço computacional. Já no modelo mesoscópico, a dificuldade da "equação íntegro-diferencial é a representação adequada do termo de interação presente nesta equação, tipo-Boltzmann, considerando a hipótese de continuidade na função distribuição” [10].

Na dinâmica de evacuação, o pânico é definido pela "quebra da ordem e do comportamento cooperativo de indivíduos devido a uma reação ansiosa provocada por um certo evento" [8]. Analogamente, Souza [11] caracteriza o pânico como uma reação psicológica em que uma ansiedade toma conta do indivíduo, que pode apresentar um comportamento de aceitação ao estímulo de desastre, levando, em último caso, a uma perda dos sentidos. "A reação psicológica causada no desastre ambiental pode fazer a capacidade adaptativa e a capacidade de resposta ao ambiente diminuírem em pouco tempo". A partir dos modelos analíticos e com a possibilidade de utilização da ferramenta computacional, desenvolveram-se novas abordagens para as reproduções dinâmicas do processo de evacuação e seu estudo [9].

\section{MODELAGEM E SIMULAÇÃO COM HUMANOS VIRTUAIS}

A visão computacional é definida como ciência ou tecnologia relacionada à visão de uma máquina. Essa definição está relacionada à forma como um computador enxerga a sua volta, captando imagens e dados multidimensionais por meio de sensores, câmeras de vídeo, scanners e outros dispositivos. Esta tecnologia abrange uma série de métodos, técnicas, equipamentos e softwares que possibilitam captar ou adquirir, processar, reconhecer, manipular e analisar os objetos que compõem uma imagem [12]. A dinâmica de pedestres é um componente importante da dinâmica de grupos e está interligada a outras áreas, tais como engenharia de tráfego, arquitetura e sócio psicologia. A simulação com humanos virtuais, nesta pesquisa, busca evidenciar aspectos da arquitetura que possam causar, retardar ou aumentar o tempo de evacuação dos locais analisados.

À princípio, o empirismo e a simples observação de situações reais servem de base para o desenvolvimento das teorias gerais e das fórmulas e parâmetros de concepção e 
operação de espaços. Com base nisso, são desenvolvidos modelos que, devidamente calibrados e validados, configuram-se em instrumentos de auxílio à concepção e operação dos espaços de grande fluxo de pessoas [3].

Com o avanço da tecnologia dos computadores, a modelagem de fluxos de pedestres tem sido amplamente utilizada e considerada como um instrumento adequado para avaliação de desempenho das construções e da qualidade das instalações para pedestres [7;13]. Os modelos de simulação podem ser classificados com base na representação do espaço (contínuo / grade com base / estrutura de rede), propósito (finalidade específica, uso geral) e o nível de detalhe (macroscópica, mesoscópica ou microscópica). Teorias e modelos focados especialmente em situações de emergência ainda estão em fase inicial de desenvolvimento, principalmente aqueles destinados ao estudo de fluxos de pedestres em espaços ao ar livre. Existem vários modelos de simulação de fluxos de pedestres e todos os sistemas de modelagem são considerados úteis. Ocorre que nenhum fornece uma gama completa de cenários em testes para fins de segurança.

Modelos Contínuos são modelagens que tem como base o fato de que a multidão é composta por indivíduos que reagem a eventos em torno dele. Esses modelos são muito utilizados para simulação da dinâmica de fluidos e gases. Como a dinâmica de pedestres possui muitas analogias com esses sistemas, existem modelos que descrevem o comportamento de pedestres utilizando equações diferenciais não lineares [14]. Um exemplo de modelo contínuo é o Modelo Forças Sociais do inglês Social Forces Model, desenvolvida em 1995 pelo Professor Dirk Helbing. Os movimentos de pedestres podem ser descritos como se fossem sujeitos a Forças Sociais. Estas forças são exercidas nos pedestres diretamente pelo ambiente. Nesse caso, os indivíduos repelem ou atraem uns aos outros como partículas atômicas, com diferentes graus de força e com base no seu próprio conforto pessoal, sendo uma medida das motivações internas dos indivíduos para realizarem determinadas ações / movimentos.

Nos modelos de pedestres que utilizam Autômata Celular, também conhecido como Partícula Hopping, o espaço é representado como uma grade simétrica, formada por células em uma condição específica. Essas modelagens são baseadas em uma matriz, onde a área ocupada por cada indivíduo é uma célula. Essas células passam de um estado para o outro de acordo com regras próprias que determinam o comportamento do sistema. As regras processam o estado de cada célula como uma função de seu estado anterior e o estado das células vizinhas em uma abrangência programada. Cada célula do sistema influencia e é influenciada pelas demais. O objetivo deste tipo de modelo é simular ambientes dinâmicos, ou seja, que mudam através do tempo e através de ciclos de interação [15;16;17]. Segundo Toyama [14], um dos modelos desenvolvidos para autômata celular é o modelo criado por Schadschneider e colaboradores (Modelo de Autômato Celular Estocástico para Simulação de Dinâmica de Pedestres). Ele é considerado extremamente eficiente, permitindo a simulação rápida de grandes multidões, reproduzindo características coletivas e de auto-organização de dinâmica de pedestres, tais como a formação de filas, fluxos em corredores largos e oscilações de fluxos encontrados em portas.

A abordagem com Multiagentes é um método computacional que permite a construção de um ambiente ocupado por indivíduos, capazes de interagir uns com os outros. Essa abordagem traz, aos modelos de pedestres, uma maneira nova de simulação entre a interação de pessoas, que não é alcançada através dos métodos tradicionais de modelagem [18]. Esta técnica de modelagem simula cada pedestre (individualmente) que se move e interage com o ambiente e com outras pessoas. Surgiu nos anos 1970, mas somente há pouco tempo conseguiu atingir seu potencial como ferramenta prática de aplicação. 


\section{MODELOS COMPUTACIONAIS DE EVACUAÇÃO DE PESSOAS EM SITUAÇÃO DE EMERGÊNCIAS}

Modelos computacionais de evacuação são ferramentas amplamente utilizadas para avaliação da segurança das pessoas em um edifício ou locais de aglomeração. Conta-se com uma grande variedade de modelos de evacuação, podendo gerar certa confusão na decisão de qual modelo adotar em determinada tarefa [19]. Contudo, cada modelo possui características únicas e capacidades diversas de simulação.

Os experimentos práticos apresentam altos custos e dados experimentais podem ser difíceis de serem captados. Por esses motivos, as simulações computacionais de evacuação têm-se tornado populares [20]. Além disso, Yang et al. [21] mencionam as preocupações éticas, e até mesmo legais, recorrentes da utilização de pessoas em experimentos de evacuação, em situação de pânico, devido aos riscos inerentes. A modelagem também é uma solução pela escassez de dados reais de evacuação e de experimentos práticos precisos [22].

Dentro dos estudos dos softwares de engenharia, desenvolvidos por meio de modelos matemáticos especiais que possibilitam o estudo de saídas de emergência em edificações estão Wagner [23], Cassol et al. [24] e Montenegro [25]. Esses softwares possuem características específicas e trabalham com a filosofia da simulação através de informações das edificações obtidas de plantas de programa CAD ou elementos geométricos. Cunha [26] afirma que, no cenário internacional, a simulação computacional já é utilizada como ferramenta de avaliação das decisões projetuais de edifícios de alto padrão ou de patrimônio histórico.

Para aprofundamento da análise dos diversos softwares disponíveis no mercado referentes à simulação do fluxo de pedestres com humanos virtuais, reúne-se alguns simuladores estudados na academia.

\subsection{EXIT89 X SIMULEX}

Em seu estudo comparativo entre dois softwares de simulação computacional de evacuação, Kuligowski e Milke [19] analisam o EXIT89 e Simulex. Eles buscam possíveis diferenças e similaridades na aplicação dos simuladores que utilizam o comportamento humano de forma implícita na modelagem. O mesmo objeto de estudos é utilizado pelos autores, um hotel de 21 andares e as escadas são o objetivo final dos ocupantes. Para os cenários, foram utilizados os dados estatísticos de casos reais de incêndio tabulados da National Fire Protection Association - NFPA.

O programa EXIT89 é descrito pelos autores como um modelo capaz de simular grandes populações que ocupam estruturas de grande altura. Ele se utiliza de uma série de nós (quartos, corredores e escadas) e arcos (distâncias entre nós). É capaz de analisar o progresso de um grande número de pessoas em um grande edifício geometricamente complexo. Ele gera edifícios bidimensionais a partir de desenhos CAD para cada andar. Outras diferenças e características são apontadas pelos autores: o tamanho das pessoas e o decrescimento da velocidade de acordo com a densidade da população, no caso do EXIT89; e tipos de ocupantes: homens, mulheres, crianças, cada um com tamanhos de corpos diferentes, bem como diferentes velocidades, no caso do Simulex.

Nos resultados das simulações obtiveram: Quanto aos tempos de evacuação, as simulações no software EXIT89 apresentaram tempos $25 \%$ a $40 \%$ mais baixos que no software Simulex. Na análise de delimitação, o EXIT89 produziu tempos de evacuação máximos 30 \% a 40 \% menores do que Simulex. Além disso, o Simulex mostrou que oferece mais possibilidades de entrada de dados de característica dos ocupantes do que o EXIT89. Este software não tem a capacidade de simular a interação entre os ocupantes, fazendo com que os ocupantes mais lentos não interfiram nos tempos de evacuação dos demais ocupantes. 
Os pesquisadores aconselham a compreensão total da funcionalidade do simulador, a fim de avaliar se os algoritmos e os métodos de movimento são ou não realistas.

\subsection{CROWDSim}

Desenvolvido pelo Laboratório de Simulação de Humanos Virtuais - VHLab PUCRS, o software CrowdSim, é uma ferramenta de simulação computacional de situações de aglomeração de pessoas em diversos ambientes, levando em conta as questões de conforto e segurança das pessoas envolvidas [27]. Esse software é o primeiro desenvolvido no Brasil, custa aproximadamente dez vezes menos do que as ferramentas internacionais e realiza análises complexas que levam em consideração diferentes situações, como eventos de pânico e emergência, incluindo a análise de diversos tipos de ocupantes - como idosos, crianças e pessoas com dificuldade de locomoção e suas reações [28].

Cassol et al. [24] aplicam a ferramenta em uma escola, desenvolvendo um serious game. A edificação a ser analisada deve ser representada em 3D e sua estrutura e acessos representados de maneira fidedigna. O programa realiza os cálculos de rota baseados em grafo. A distância entre dois contextos são arestas, enquanto o ponto de ligação entre os contextos é considerado um nodo. Com os dados de saída do software é possível avaliar a densidade populacional ao longo do tempo para as diversas áreas do local estudado, o tempo total de evacuação, a trajetória percorrida pelos agentes (ocupantes) e os níveis de concentração, expressos em mapas de densidade. A partir dessas análises tem-se a indicação de gargalos e pontos críticos, a fim de buscar soluções para prevenção e correção desses eventos indesejáveis [27].

\subsection{VHD++}

Ulicny e Thalmann [29] utilizam a reconstrução em três dimensões de locais antigos e complexos, somados à implementação de humanos virtuais com o maior realismo possível, incluindo aspectos visuais e acústicos desses patrimônios reconstruídos graficamente. Os autores utilizam o software de simulação de multidões chamado VHD++, que é um programa computacional em tempo real, sendo possível carregar essas informações para a realidade virtual, incluindo o comportamento de seres humanos virtuais e informações do local como o espaço em si, localização de portas, entre outros. Geram-se "sequências de quadros codificados de animação corporal em tempo real ou pré-gravadas, caminhando para uma localização particular usando o modelo de movimento ambulante ou tocando um som” [29]. Os autores simularam uma mesquita virtual durante a oração matutina com uma população de fiéis.

Foi utilizado o software de modelagem Studio Max. Nele pode-se observar "sistema de comportamento de regras que permite a representação flexível de um cenário complexo de forma relativamente fácil de se adaptar a diferentes edifícios ou números diferentes de pessoas” [29].

\subsection{LEGION}

Legion é um software de simulação dinâmica baseado em um modelo multiagente utilizado pelas consultorias Steer Davies Gleave e Modelle Logística e Engenharia para modelagem e simulação de fluxos de pedestres. Permite o desenvolvimento de algoritmos para distintas situações e contextos de simulação de pedestres, como para áreas de trabalho, lazer, situações de emergência, entre outros. Cada pedestre é modelado como uma unidade autônoma, que se move em um espaço contínuo em intervalo de tempo de 0,6 segundos. $\mathrm{O}$ software permite a construção dos espaços arquitetônicos, com importação direta do formato CAD e permite a modelagem de elementos de circulação, tais como esteiras rolantes, escadas, rampas, elevadores, entre outros [30]. 
De acordo com o modelo, as decisões de navegação tomadas pelos pedestres ocorrem em três níveis: estratégica, relacionada à escolha do destino; tática, relativa à macronavegação; e operacional, relativa à micronavegação. A escolha do destino está relacionada à determinação do objetivo do pedestre. Na macronavegação, com base na geometria do espaço a ser percorrido, o pedestre irá encontrar a rota mais curta a fim de que realize o menor esforço possível dentro dos fatores de satisfação apresentados. A micronavegação está relacionada ao comportamento de cada pedestre em mudar sua trajetória devido a fatores específicos, tais como, a necessidade de desvio de obstáculos, etc. [30]. Para calibração e determinação de perfil de pedestres, o software utiliza base de perfis populacionais selecionáveis que variam conforme a região do mundo. Os perfis são determinados por uma série de parâmetros, entre eles a velocidade de deslocamento e o espaço que o pedestre ocupa. Neste software, as características relacionadas ao comportamento humano são mais restritas.

\subsection{PTV VisWalk E PTV Vissim}

O software PTV Viswalk tem como objetivo analisar a capacidade e o uso eficiente dos espaços do objeto de estudo, através da simulação de movimentação de pedestres, em ambientes internos e externos. O software é comercializado como um módulo do PTV Vissim, do alemão Verker In Stadten-SIMmultation, ou simulação de tráfego na cidade (tradução dos autores), que possui flexibilidade de opções e parâmetros e objetiva a utilização de simulações de pedestres em situações complexas envolvendo grande número de usuários. O software além de ser utilizado no estudo de evacuação de edifícios, permite trabalhar com simulação de deslocamentos de pedestres em situações cotidianas em duas ou três dimensões, além de possuir módulos para microssimulação de automóveis [30]. Os softwares são desenvolvidos por uma empresa sediada em Karlsruhe, Alemanha, a PTV Planung Transport Verkehr AG PTV Group que trabalha com inovações nas áreas de mobilidade urbana, logística e engenharia de trânsito. O simulador tem como características principais, a possibilidade de detectar pontos de estrangulamentos e possíveis obstáculos que podem oferecer potenciais riscos de lentidão na evacuação dos locais. Além disso, utiliza variáveis do comportamento humano que, por exemplo, evitam a colisão entre os indivíduos em uma movimentação normal e obstáculos, a partir de um estudo de pedestres em contra fluxo.

A simulação pode atuar tanto na fase de elaboração para novos projetos, como na avaliação das condições de esvaziamento de edificações existentes. Os resultados permitem uma visualização integral de ambientes e não tem capacidade limite em número de indivíduos para realizar a simulação [31]. A ferramenta está baseada em análise microscópica para simulação de pedestres e, dentre suas aplicações, encontra-se o plano de evacuação de edifícios e eventos especiais, no qual pode ser estudado: a segurança em locais públicos; a avaliação de medidas estruturais e organizacionais destinadas a reduzir e controlar o comportamento das pessoas em situações de emergência; os perigos potenciais e o planejamento de fluxos de pedestres em edifícios, estádios e outras instalações; a simulação de rotas de saída e cenários de evacuação em edifícios altos e túneis.

$\mathrm{Na}$ análise da capacidade de simulações de cada programa computacional, pôde-se perceber um recente avanço tecnológico nos simuladores computacionais. Com o uso desses softwares pode-se realizar um estudo seguro e de baixo custo nos objetos de estudo propostos.

\section{APLICAÇÃO DO SOFTWARE DE SIMULAÇÃO COMPUTACIONAL}

Dentre os softwares apresentados fez-se a avaliação de qual utilizar para a aplicação neste estudo. Todos se mostraram adequados quanto ao gráfico avançado e à utilização de modelagens em três dimensões, o que possibilita uma avaliação mais ampla dos cenários a 
serem estudados. Inclusive, todos utilizam como parâmetro de caracterização dos ocupantes o comportamento humano, medida essencial na avaliação da evacuação de pessoas em situação de emergência.

A disponibilidade do software com grande confiabilidade dos resultados, a simulação atuando tanto na fase de elaboração para novos projetos, como na avaliação das condições de esvaziamento de edificações existentes conduziu, nesta pesquisa, a adoção do software PTV Viswalk. Além disso, foi disponibilizada pelo grupo PTV, detentor dos direitos, uma licença acadêmica para os autores. A licença viabiliza a utilização de todos os recursos do software e não apenas os oferecidos na versão trial cedido por trinta dias. O grupo também estende aos acadêmicos um suporte via site e e-mail, além de manuais, o que permite a troca de informações e possíveis dúvidas que surjam ao longo da pesquisa. Com isso exposto tem-se a aplicação do software computacional nos modelos em três dimensões criados para este estudo. As modelagens foram construídas a partir dos projetos em formato digitais dos três edifícios da cidade de Florianópolis / Brasil e essas representam os espaços internos e externos que compreendem os ambientes onde se concentram os ocupantes de cada edifício, os caminhos de circulação existentes em cada prédio, suas saídas e obstáculos presentes nesse caminho.

Com estes modelos foram rodados os cenários das simulações para verificar o comportamento dos pedestres durante as evacuações de emergência. O simulador da PTV permite que a modelagem dos pedestres possa ser escolhida entre as abordagens car following model do Professor Wiedemann, em que os pedestres são modelados como um tipo de veículo e não se movem livremente, mas sim ao longo de determinados pontos pré-definidos, ou a abordagem Modelo Forças Sociais, que permite aos pedestres andarem independentemente do seu destino, sem um modelo de rede pré-definido das suas trajetórias. O Professor Helbing é consultor científico da PTV Group e ele, especialmente, expandiu o modelo para uso no software Viswalk [32]. Para o desenvolvimento deste trabalho foi escolhido o modelo Social Forces Model devido à simulação apresentar resultados mais realistas.

\subsection{CARACTERÍSTICAS GRÁFICAS DO SOFTWARE}

O modelo no software PTV Vissim pode ser visualizado em duas ou três dimensões, ou dos dois modos simultaneamente. Para a captação dos mapas de densidade é utilizada a visualização 2D, já para a análise visual dos pontos de gargalo, interação entre as pessoas e como elas se comportam no ambiente de evacuação, é feita a visualização em 3D durante a execução da simulação. Posiciona-se os ocupantes desses edifícios em cada ambiente. Na planta 2D do software essa população é representada por um ponto preto com borda azul, como cor padrão. É necessário estipular em cada área a quantidade de pessoas, com a definição de decisão que se encontra ali. Também é determinado a decisão de deslocamento. É definido então a origem e o destino dos pedestres. A origem, representada por um ponto laranja, é aplicada no ambiente de onde o grupo de pessoas irá partir e o destino, ponto azul claro, posicionado no destino dos ocupantes naquela simulação. Também podem ser definidos caminhos obrigatórios para os pedestres percorreram. As áreas verdes são as áreas de origem dos deslocamentos e as em vermelho as áreas de destino dos pedestres. As demais áreas, escadas e rampas são representadas na cor cinza e são utilizadas pelos pedestres nos deslocamentos. Todos os obstáculos são representados na cor bordô e podem possuir alturas diferentes: as paredes construídas na altura de 1,50m possibilitam a visualização dos pedestres durante a evacuação, já os demais obstáculos como mobiliário, guarda-corpos e outros, em menos dimensão.

\subsection{COMPOSIÇÃO DOS PEDESTRES}

A adoção para a composição dos pedestres é iniciada pelo tipo de pedestre, sendo eles: Homens, mulheres, mulheres e crianças e cadeirantes. A velocidade de distribuição 
desejável é escolhida. Para esse estudo foram utilizadas as velocidades de $1,2 \mathrm{~m} / \mathrm{s}$ para as situações de saída normal das edificações e de $1,53 \mathrm{~m} / \mathrm{s}$ para as situações de saída em situação de emergência. Os avatares possuem variação nas dimensões e nas cores que compõe as suas aparências. O comportamento do deslocamento na rede é definido de acordo com o padrão do software, destacando-se neste: o tempo de inércia, tau $=0,4 \mathrm{~s}$, que pode ser relacionado a um tempo de reação a emergência; ReactToN $=8$, que são 8 os pedestres mais próximos que exercem influência sobre cada pedestre em seu deslocamento; e noise $=1,2$ que quanto maior este valor, mais forte as forças aleatórias que são adicionadas se um pedestre permanece abaixo de sua velocidade desejada por um certo tempo.

\subsection{EXTRAÇÃO DE DADO}

Para cada simulação rodada, opta-se pelos dados que se deseja obter nas configurações de avaliações. Os dados externos são gerados em arquivos do programa PTV que podem ser importados para planilha eletrônica para fazer as leituras e análises necessárias.

\subsection{MODELAGEM}

As duas formas de importação de modelos permitida pelo software são: através de arquivo BIM da sigla de Building Information Modelling, ou Modelagem da Informação da Construção, e plantas em 2D do AutoCAD. Foram inicialmente construídas a modelagem em três dimensões utilizando o software Revit, porém na importação algumas informações como escadas e rampas não foram lidas. Utilizou-se então a construção da modelagem 3D a partir das plantas do software Auto CAD. Nesse modo, faz-se a importação dos pisos planos, paredes e demais obstáculos, contudo a construção das escadas e rampas deve ser feita dentro do PTV. Observou-se que layouts específicos, com piso inclinado que são início o final de trajeto de pedestres, obstáculos no início e fim de escadas e rampas (Figura 1), entre outros detalhamentos diferenciados inviabiliza a execução de simulações nesses ambientes. Enquanto isso, plantas de layout retangulares e mais simplificados atendem aos requisitos do software para as simulações, pois nesses casos não ocorrerão possíveis discrepâncias de resultados devido a essas características de layout.

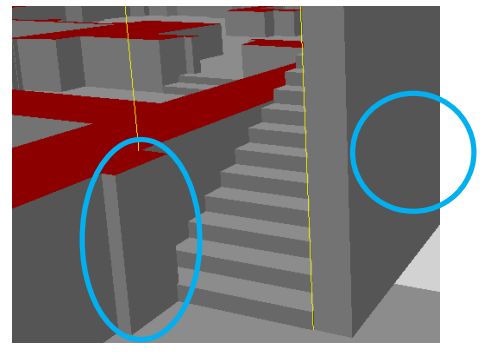

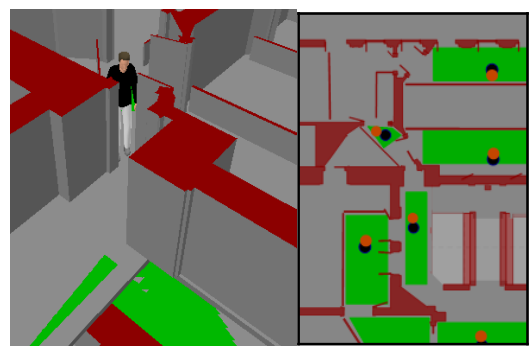

Figura 1: Obstáculo no final da escada não permite a execução da simulação.
Figura 2: Avatar preso no obstáculo folha da porta e a alteração do obstáculo.

Também foram necessárias alterações em mobiliário e em espaços reduzidos para que as simulações não apresentassem erros. Percebeu-se avatares presos na abertura da folha de portas, por exemplo (Figura 2). Numa situação real as pessoas passam de lado nesse ponto da edificação, porém os avatares andam para a frente, sem virar o torço, o que faz com que fiquem presas nesse ponto durante a simulação. 


\subsection{RESULTADO DAS SIMULAÇÕES}

Além da análise dos dados extraídos das simulações pode-se fazer análise visual das simulações enquanto essas ocorrem. Os edifícios estudados possibilitaram a visualização dos pedestres em situação de abandono dos edifícios tanto em mesmo nível, quando em mais pavimentos.

A análise visual das simulações possibilitou a observação da formação de gargalos, onde há o estrangulamento da circulação, ou seja, onde esta tem suas dimensões reduzidas (Figura 3). Também foram possíveis as visualizações das interações entre pedestres em áreas de escadas. Os pedestres encaminham-se a saída de emergência configurada para o cenário de simulação e a sua velocidade diminui consideravelmente quando se aproxima das escadas mais solicitadas, ou seja, aquelas em que um maior número de pedestres se desloca a mesmo tempo. Isso provoca uma aglomeração junto a essas escadas que por sua vez, causa um retardamento do abandono das salas mais próximas (Figura 4). Não são visíveis esmagamentos ou pisoteamento, uma vez que os avatares permanecem na posição de caminhada, movimentando-se sem sair do lugar, como uma força de avanço a rota traçada, porém obstruída. Alguns pedestres escolhem um caminho mais longo, desviando das rotas que se encontram aglomeradas.

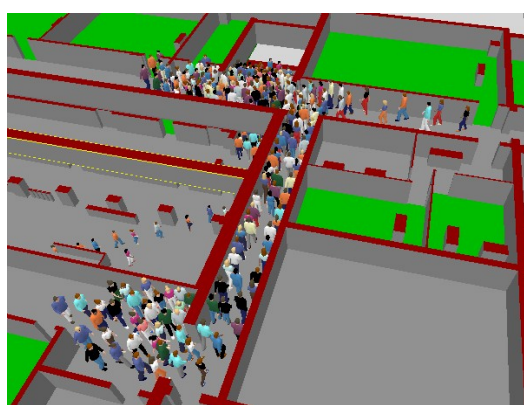

Figura 3: Gargalos e aglomeração junto a escada.

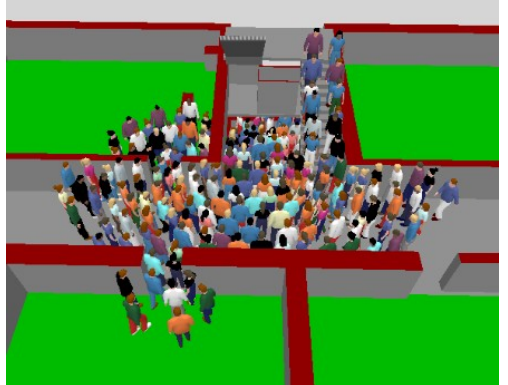

Figura 4: Aglomeração e retardamento do abandono das salas.

Quando utilizado o cenário em que a saídas de emergência mais próximas encontram-se bloqueadas e há apenas uma possibilidade de saída, as aglomeração e baixa velocidade de deslocamento ficam ainda mais intensificadas da metade para o final da simulação, período em que grande número os ocupantes do edifício estão em direção a uma única saída (Figura 5).

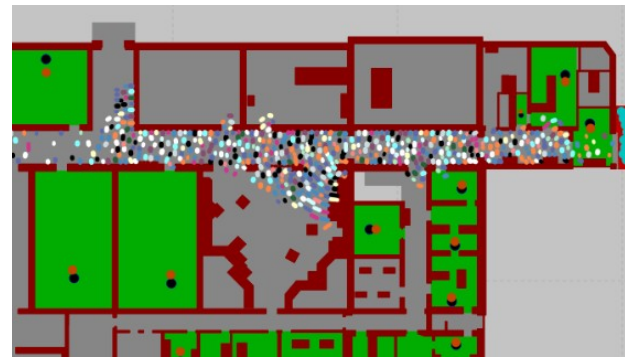

Figura 5: Cenário com uma única saída.

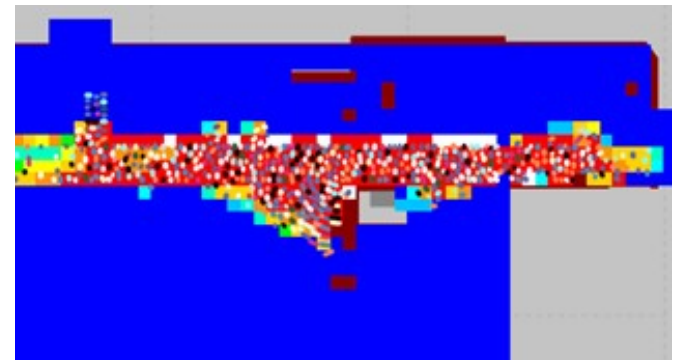

Figura 6: Mapa de densidade - cenário com única saída.

A Figura 6, representa um momento crítico desse cenário onde a maior parte do pavimento térreo em que se encontram pessoas, esses concentram-se em um número igual ou maior que 5 pedestres por $\mathrm{m}^{2}$, áreas em vermelho, de acordo com o esquema de cores de 
densidade de Weidmann [30], no momento $250 \mathrm{~s}$ da evacuação, e existem na rede 670 ocupantes que ainda não deixaram o prédio.

\section{CONSIDERAÇÕES FINAIS}

O estudo da evacuação segura de pessoas em situação de incêndio e em outras emergências tem o objetivo principal de salvar vidas. Esse artigo teve o objetivo estudar a movimentação de pedestres durante evacuação de emergência. Para isso foi utilizado o simulador computacional PTV Viswalk em edificações existentes. A revisão da literatura realizada elucidou as questões referentes ao estudo de tráfego de pedestres, a modelagem e simulação com humanos virtuais e os modelos computacionais de evacuação de pessoas em situação de emergência que existem atualmente. Com isso pôde optar pelo software da PTV para realizar a simulação computacional de evacuação de pedestres em situação de emergência. Durante o processo da pesquisa, observou-se dificuldades na manipulação dos softwares que ainda precisam ser mais investigadas e solucionadas, como foi o caso dos arquivos convertido a partir do arquivo IFC e que levou a impossibilidade de realizar o estudo completo em um dos edifícios estudados.

Entende-se que a adoção de método computacional avançado baseado em desempenho, como foi feito por meio do estudo de simulação computacional, promove um maior conhecimento do edifício e de como as pessoas se comportam nesses ambientes específicos, em situações de abandono. Essa ferramenta promove a melhor análise do edifício, pois é possível visualizar o que ocorre em possíveis situações de emergência.

Entende-se que a simulação realizada nestas plantas diferenciadas, construídas de forma simplificada para a execução das simulações descaracteriza o edifício particular e traz resultados irreais comprometendo estudo que levam em conta peculiaridades construtivas, como é o caso de edificações existentes. Com a construção da modelagem realizada e com o conhecimento dos erros ocorridos, é possível a busca pela solução em um trabalho futuro, para então possibilitar a execução da simulação computacional de evacuação para este objeto de estudos. Os simuladores computacionais podem ser excelentes ferramentas para a análise dos projetos pelo Corpo de Bombeiros, pois amplia a avaliação das condições de segurança quanto a evacuação segura de pedestres para além dos aspectos normativos. Acredita-se que num futuro próximo essa ferramenta seja efetivamente utilizada para essa finalidade

\section{Agradecimentos}

O presente trabalho foi realizado com apoio da Coordenação de Aperfeiçoamento de Pessoal de Nível Superior - Brasil (CAPES). Os autores também agradecem ao Grupo PTV pela licença estudantil concedida do software PTV Viswalk.

\section{REFERÊNCIAS BIBLIOGRÁFICAS}

[1] MARTINS; PONTES; KARAN; FINCO. O Perigo das multidões. Revista Época. 2013. Disponível em: http://revistaepoca.globo.com/tempo/noticia/2013/02/o-perigodas-multidoes.html. Acesso em: 28 mai. 2019.

[2] NAGAI, Ryoichi; NAGATANI, Takashi; ISOBE, Motoshige; ADACHI, Taku. Effect of exit configuration on evacuation of a room without visibility. Physica A: Statistical Mechanics and its Applications, v. 343, p. 712-724, 2004. https://doi.org/10.1016/j.physa.2004.06.061. 
[3] DAAMEN, Winnie; HOOGENDOORN, Serge. Capacity of doors during evacuation conditions. Procedia Engineering, v. 3, p. 53-66, 2010. https://doi.org/10.1016/j.proeng.2010.07.007.

[4] CAMPOS, Vania; BANDEIRA, Renata; BANDEIRA, Adriano. A method for evacuation route planning in disaster situations. Procedia-Social and Behavioral Sciences, v. 54, p. 503-512, 2012. https://doi.org/10.1016/j.sbspro.2012.09.768.

[5] FAHY, Rita F.; PROULX, Guylene; AIMAN, Lata. Panic'and human behaviour in fire. National Research Council Canada, 2009.

[6] VORST, Harrie C. M. Evacuation Models and Disaster Psychology. Procedia Engineering, 3, p. 15-21. 2010. https://doi.org/10.1016/j.proeng.2010.07.004.

[7] HELBING, D; FARKAS, I; VICSEK, T. Simulation of pedestrian crowds in normal and evacuation situations. Pedestrian and evacuation dynamics, v. 21, n. 2, p. 21-58, 2002.

[8] HELBING, Dirk; JOHANSSON, Anders. Pedestrian, crowd, and evacuation dynamics. arXiv preprint arXiv:1309.1609, 2013.

[9] KHOLSHEVNIKOV, Valerii V.; SAMOSHIN, Dmitrii A. Movement regularities of pedestrian flow - basics for evacuation modelling and management. Resilience of Cities to Terrorist and other Threats, Moscou, 2008.

[10] VARGAS, Marina; GRAMANI, Liliana Madalena; KAVISKI, Eloy; BALBO, Fábio André. Modeling the flow of pedestrians by the macroscopic theory. Revista Brasileira de Ensino de Física, v. 34, n. 4, p. 1-10, 2012.

[11] SOUZA, João Carlos. Emergências em locais com reunião de grande público: o papel da logística humanitária. XXIX Congresso Nacional de Pesquisa em Transporte da ANPET, Ouro Preto, 2015.

[12] MILANO, Danilo de; HONORATO, Luciano Barrozo. Visão computacional. Universidade Estadual de Campinas, p. 1-7, 2010.

[13] OSARAGI, Toshihiro. Modeling of Pedestrian Behavior and Its Applications to Spatial Evacuation. In: International Joint Conference on Autonomous agents and multiagents systems, v. 2, p. 836-843, 2004.

[14] TOYAMA, Marcelo Costa. Uma Abordagem Multiagentes para Dinâmica de Pedestres. 2006. Dissertação (Mestrado em Computação) - Instituto de Informática, Universidade Federal do Rio Grande do Sul, Porto Alegre, 2006.

[15] DIJKSTRA, J.; TIMMERMANS H. J. P.; JESSURUN A. J. Multi-Agent Cellular Automata System for Visualising Simulated Pedestrian Activity. Fourth International Conference on Cellular Automata for Research and Industry, Berlim, 2000.

[16] KLÜPFEL, Hubert Ludwig. A cellular automaton model for crowd movement and egress simulation. 2003. Tese (Doutorado em Ciências), Universidade Duisburg Essen, Duisburg, 2003.

[17] WAS, Jaroslaw. Cellular Automata model of pedestrian dynamics for normal and evacuation conditions. Intelligent Systems Design and Applications - ISDA, Warsaw, 2005. 
[18] TEKNOMO, Kardi; GERILLA Gloria P. Sensitivity Analysis and Validation of a Multi-Agents Pedestrian Model. Journal of the Eastern Asia Society for Transportation Studies EASTS, v. 6, p. 198-213, 2005. https://doi.org/10.11175/easts.6.198.

[19] KULIGOWSKI, Erica D.; MILKE, James A. A Performance-Based Design of a Hotel Building Using Two Egress Models: A Comparison of the Results. Human Behaviour in Fire Symposium. p. 399-410, 2004.

[20] SHI, Jianyong; REN, Aizhu; CHEN, Chi. Agent-based evacuation model of large public buildings under fire conditions. Automation in Construction, v. 18, n. 3, p. 338-347, 2008. https://doi.org/10.1016/j.autcon.2008.09.009.

[21] YANG, L.Z.; ZHAO D.L.; LI J.; FANG T.Y. Simulation of the kin behavior in building occupant evacuation based on cellular automaton. Building and Environment, v. 40, n. 3, p. 411-415. 2005. https://doi.org/10.1016/j.buildenv.2004.08.005.

[22] LEIJONMARCK, Eric; OLERGÅRD, Thomas. Modelling of panicking pedestrians during emergency evacuation. 2013. Thesis (Bachelor of Science) - Royal Institute of Tecnology, 2013.

[23] WAGNER, Robson. Projeto para saídas de emergência: o conceito de desempenho em Santa Catarina. 2008. 119 f. Dissertação (Mestrado em Arquitetura e Urbanismo) Universidade Federal de Santa Catarina, Florianópolis. 2008.

[24] CASSOL, Vinícius Jurinic; BRASIL, Jovani Oliveira; Neto; AMYR B. Fortes; BRAUN, Adriana; MUSSE, Soraia Raup. An approach to validate crowd simulation software: a case study on crowdsim. In Proceedings of SBGames 2015 - XIV SBGames. Teresina, 2015.

[25] MONTENEGRO, Mariana Lima Oliveira. Análise de desempenho das saídas de emergência por meio de simulações computacionais: o caso de projetos de edifícios universitários. 2016. 175f. Dissertação (Mestrado em Arquitetura e Urbanismo) Centro de Tecnologia, Universidade Federal do Rio Grande do Norte, Natal, 2016.

[26] CUNHA, Leonardo Jorge Brasil de Freitas. O desempenho da compartimentação horizontal seletiva na promoção da segurança contra incêndio em edificações. 2016. 238f. Tese (Doutorado em Arquitetura e Urbanismo) - Centro de Tecnologia, Universidade Federal do Rio Grande do Norte, Natal, 2016.

[27] CASSOL, Vinícius Jurinic; RODRIGUES, Rafael Araújo; CARNEIRO, Luiz Carlos Cunha; SILVA, Anderson; MUSSE, Soraia Raup. CrowdSim: Uma ferramenta desenvolvida para simulação de multidões. In: I Workshop de Simulação Militar SBGames, 2012.

[28] SOFTWARE simula comportamento de multidões em grandes eventos para evitar incidentes. Finep - Financiadora de Inovação e Pesquisa, Rio de Janeiro, 2013. Disponível em: http://finep.gov.br/noticias/todas-noticias/3653-software-simulacomportamento-de-multidoes-em-grandes-eventos-para-evitar-incidentes. Acesso em: 3 out. 2017.

[29] ULICNY, Branislav; THALMANN, Daniel. Crowd Simulation for Virtual Heritage. In: Proceedings first international workshop on 3D, Geneva, p. 28-32, 2002.

[30] TERRA, Ulisses Demarchi Silva. Arquitetura em espaços de fluxo: modelagem e simulação em estações metroferroviárias e espaços de multidão. 2014. Dissertação 
(Mestrado em Arquitetura e Urbanismo) - Faculdade de Arquitetura e Urbanismo, Universidade de São Paulo, São Paulo, 2014.

[31] PTV Group. Brochura do Viswalk. 2017. Disponível em: http://visiontraffic.ptvgroup.com/fileadmin/files_ptvvision/Downloads_N/0_General/2_Products/ 3_PTV_Viswalk/EN-UK_PTV_Viswalk_Brochure.pdf. Acesso em: 03 out. 2017.

[32] PTV Group. PTV Vissim User Manual. Karlsruhe, Germany. 2018. 